\title{
Quantum Critical Behavior with Massless Staggered Fermions in Three Dimensions
}

\author{
Shailesh Chandrasekharan ${ }^{* \dagger}$ \\ Department of Physics, Duke University, Durham, North Carolina 27708 \\ E-mail: schephy.duke.edu
}

\begin{abstract}
We report on studies of quantum critical behavior in three dimensional lattice Gross-Neveu models with one flavor of staggered fermions. We focus on two models, one with $S U(2) \times Z_{2}$ symmetry and the other with an $S U(2) \times U(1)$ symmetry. Both these models could not be studied earlier with conventional Monte Carlo methods due to sign problems. However, the fermion bag approach is free of sign problems for these models and allows us to compute the critical exponents at the quantum phase transition that separates the massless fermion phase at small couplings and the massive fermion phase at large couplings. Our results help resolve some old puzzles in the field.
\end{abstract}

31st International Symposium on Lattice Field Theory - LATTICE 2013

July 29 - August 3, 2013

Mainz, Germany

\footnotetext{
${ }^{*}$ Speaker.

${ }^{\dagger}$ This talk is based on work done in collaboration with Anyi Li.
} 


\section{Introduction}

Quantum phase transitions that separate a massless fermion phase from a massive one are of general interest in particle physics [1]. While in $3+1$ dimensions interesting transitions usually require the presence of non-Abelian gauge fields [2], in $2+1$ dimensions one expects a rich class of transitions even with four-fermion interactions, since they are known to become renormalizable non-perturbatively $[3,4]$. Recent experiments in graphene have triggered renewed interest in the field [5, 6]. While many Monte Carlo calculations have been performed to compute critical properties of these phase transitions using lattice techniques, the results are far from ideal. Difficulties arise from two main sources: Firstly, fermion Monte Carlo algorithms are known to scale badly with system size, especially when fermions are massless. Most calculations are performed with a finite fermion mass which acts as another infrared regulator in addition to the system size. The presence of these two infrared scales creates difficulties in the analysis. Secondly, many fermion field theories suffer from unsolved sign problems and Monte Carlo methods become inapplicable. It should be noted here that sign problems can arise even in the absence of chemical potentials in fermion systems. Sign problems depend on the variables used to expand the partition function as a sum over classical configurations. While the chemical potential is one source of sign problems, there can be other sources. The motivation for the current work is to resolve some puzzles in previous Monte Carlo results that arise due to these sign problems.

In order to appreciate some of the puzzles in the literature, consider lattice formulations of four-fermion field theories using massless staggered fermions [7]. In three space-time dimensions a single staggered flavor produces two flavors of four-component Dirac fermions. The free fermion action for such a theory is known to be invariant under a $U(4)$ flavor symmetry. In the continuum it is possible to introduce a four-fermion interaction that preserves this symmetry fully. This is the well known continuum Gross-Neveu model [8]. On the other hand, a class of lattice GrossNeveu models constructed with a single flavor of staggered fermions break the $U(4)$ symmetry to a $S U(2) \times Z_{2}$ subgroup. Such a model was constructed and studied using Monte Carlo methods long ago [9]. In both models, as the four-fermion coupling is increased, one finds a critical point at which parity (which includes the $Z_{2}$ symmetry in the lattice model) breaks spontaneously and fermions acquire a mass. Surprisingly, the results for the critical exponents found in the continuum model match those of the lattice model although the symmetries of the two models are different. In particular both models give $v \approx 1.0$ and $\eta \approx 0.75$. While the matching of the two calculations is quoted as a success of universality, the question why the lattice interactions that break the continuum symmetries are irrelevant, is never addressed. Recently, we noted that the lattice model actually suffers from a sign problem which was ignored [10]. We will argue here that the results of the lattice calculations appear to be incorrect.

The second puzzle concerns another class of four-fermion models called Thirring models. In the continuum, with two flavors of four-component Dirac fermions, $U(4)$ flavor symmetric Thirring models have been studied recently [11]. On the other hand, a lattice Thirring model containing a single flavor of staggered fermions, with an $S U(2) \times U(1)$ symmetry, has been studied with Monte Carlo calculations for many years [12, 13, 14]. Fortunately, these lattice calculations do not suffer from sign problems and so should be reliable. While in the continuum model it was found that $v \approx 2.4$ and $\eta \approx 1.4$, on the lattice the same exponents were found to be $v \approx 0.85$ and $\eta \approx 0.65$. 
This disagreement is not surprising since it can be attributed to the difference in the underlying symmetries of the two models. However, it does raise the question if lattice calculations have uncovered a new universality class in Thirring models? On the other hand the lattice Thirring model has the same symmetries as the lattice Gross-Neveu model and so a natural question is if the critical exponents found match those of the lattice Gross-Neveu model. Since most Gross Neveu models studied so far give $v \approx 1.0$ [15], the folk lore until now was that the lattice Thirring model and the lattice Gross-Neveu model belong to different universality classes, even though their symmetries are identical. Unfortunately, the lattice Gross-Neveu model with $S U(2) \times U(1)$ symmetry could not be studied due to sign problems, and the folk lore had remained unconfirmed so far. Here we find evidence that the folk lore is incorrect and that the two models belong to the same universality class.

While both puzzles discussed above could not be resolved earlier due to sign problems in the conventional approach, the recently developed fermion bag approach can help resolve them since it does not suffer from sign problems [16].

\section{Lattice Gross-Neveu Models}

Lattice Gross-Neveu models are usually formulated with auxiliary fields that couple to fermion mass terms. In a popular formulation auxiliary fields live at the center of cubes and couple to mass terms at the corners [7]. In another class of formulations, auxiliary fields also live on the lattice sites, but couple to fermion mass terms on the neighboring sites [9]. The auxiliary field approach is natural for conventional Monte Carlo methods since the action is quadratic in fermion fields. However, with one flavor of staggered fermions it turns out that the determinant of the fermion matrix is not guaranteed to be positive. This leads to sign problems in the conventional approach. Note that the sign problem here is not due to the introduction of a chemical potential like in QCD, but simply because the mass terms have a fluctuating sign that depends on the auxiliary field.

Fortunately, we can integrate out the auxiliary fields and obtain a general four-fermion action of the form

$$
S=\sum_{x, y} \bar{\chi}_{x} D_{x y} \chi_{y}-\sum_{\langle x y\rangle} U_{\langle x y} \bar{\chi}_{x} \chi_{x} \bar{\chi}_{y} \chi_{y}
$$

where $\bar{\chi}_{x}, \chi_{x}$ denote staggered fermion fields at the lattice site $x$ and $D$ is the free massless staggered fermion matrix [17]. The four-fermion interactions $U_{\langle x y\rangle}$ usually come in four types (see Fig. 1): (1) link bonds $\mathbb{L}$ (where $x, y$ are nearest neighbor sites), (2) face bonds $\mathbb{F}$ (where $x, y$ are sites diagonally across faces of squares), (3) body bonds $\mathbb{B}$ (where $x, y$ are sites diagonally across the bodies of cubes) and (4) next-nearest-neighbor bonds $\mathbb{N}$ ( where $x, y$ are next nearest neighbor sites).

The symmetries of the lattice models can be easily determined from (2.1). When the fourfermion couplings only couple even sites with odd sites then the well known $U(1)$ chiral symmetry of staggered fermions remains unbroken. On the other hand whenever there exist couplings between even sites or odd sites the chiral symmetry is broken to the $Z_{2}$ subgroup. In addition to the chiral symmetry, there is an $S U(2)$ flavor symmetry as pointed out in [18]. Assuming the interactions couple even sites only with odd sites, it is easy to verify that the action is invariant under the 

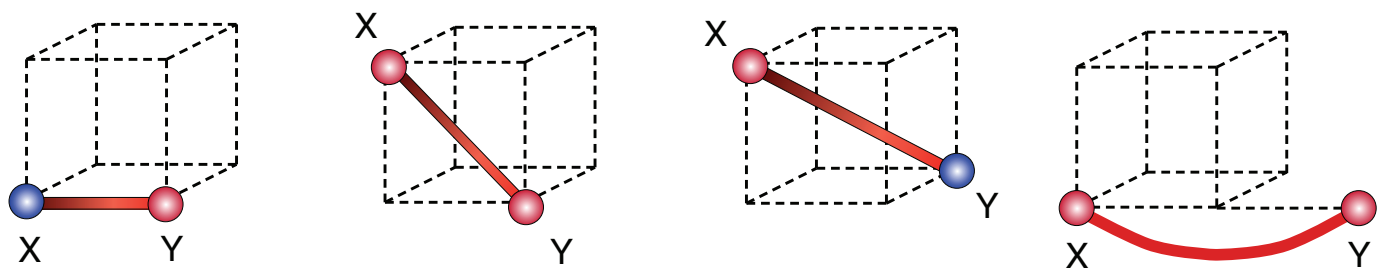

Figure 1: A pictorial representation of four-fermion couplings $U_{\mathbb{L}}$ (left), $U_{\mathbb{F}}$ (center left), $U_{\mathbb{B}}$ (center right) and $U_{\mathbb{N}}$ (right) discussed in the text. Each bond refers to four-fermion interactions of the form $\bar{\chi}_{x} \chi_{x} \bar{\chi}_{y} \chi_{y}$.

following $S U(2) \times U(1)$ symmetry,

$$
\left(\begin{array}{l}
\chi_{e} \\
\bar{\chi}_{e}
\end{array}\right) \rightarrow \mathrm{e}^{i \theta} V\left(\begin{array}{l}
\chi_{e} \\
\bar{\chi}_{e}
\end{array}\right),\left(\begin{array}{ll}
\bar{\chi}_{o} & \chi_{o}
\end{array}\right) \rightarrow\left(\begin{array}{ll}
\bar{\chi}_{o} & \chi_{o}
\end{array}\right) V^{\dagger} \mathrm{e}^{-i \theta},
$$

where the subscripts $e$ and $o$ refer to even and odd sites and $V$ is an $S U(2)$ matrix. When couplings within even sites or within odd sites are introduced then $\theta=\pi / 2$ is the only allowed value for the symmetry transformation and the action is only invariant under an $S U(2) \times Z_{2}$ symmetry.

The physics described by models with action (2.1) is simple but interesting. They all have a massless fermion phase at small couplings $U$ due to the fact that four-fermion couplings are irrelevant perturbatively. However, as the coupling increases, a second order phase transition to a massive fermion phase accompanied by spontaneous breaking of chiral symmetries occurs at a critical coupling $U_{c}$. The critical properties of this phase transition are the main focus of our studies. In the broken phase $\langle\bar{\chi} \chi\rangle \neq 0$, which means either the $Z_{2}$ or the $U(1)$ chiral symmetry is spontaneously broken depending on the symmetries of the lattice model. On the other hand the $S U$ (2) flavor symmetry remains unbroken since. It is easy to verify that the chiral condensate is a singlet under the $S U$ (2) flavor group. The critical properties of the phase transition are the main focus of studies since, as we have explained above, there are many puzzles associated with them.

\section{Results}

We have recently studied two models one with a $S U(2) \times Z_{2}$ symmetry and the other with a $S U(2) \times U(1)$ symmetry. These models were obtained from models with auxiliary fields at the center of a cubes coupled to fermions at the corners through a single coupling $U$. On integrating out the auxiliary fields, for the $Z_{2}$ model we find $U_{\mathbb{L}}=2 U_{\mathbb{F}}=4 U_{\mathbb{B}} \equiv U, U_{\mathbb{N}}=0$, while for the $U(1)$ model we find $U_{\mathbb{L}}=4 U_{\mathbb{B}} \equiv U, U_{\mathbb{F}}=U_{\mathbb{N}}=0$ [10]. Note that the model studied in Ref. [9] has $U_{\mathbb{F}}=2 U_{\mathbb{N}}=U, U_{\mathbb{L}}=U_{\mathbb{B}}=0$, while the lattice Thirring model studied in Ref. [12, 14] has $U_{\mathbb{L}}=U$, $U_{\mathbb{F}}=U_{\mathbb{B}}=U_{\mathbb{N}}=0$.

In the fermion bag method it is possible to solve (2.1) directly without introducing auxiliary fields for positive values of $U_{\langle x y\rangle}$, since the sign problem is completely solved. Further, we can work directly in the massless limit. This helps greatly in the analysis of our data. It is interesting to note that there is an important difference between spontaneous breaking of $Z_{2}$ and $U(1)$ symmetries, the former does not produce massless Goldstone bosons in the broken phase while the latter does. It is 


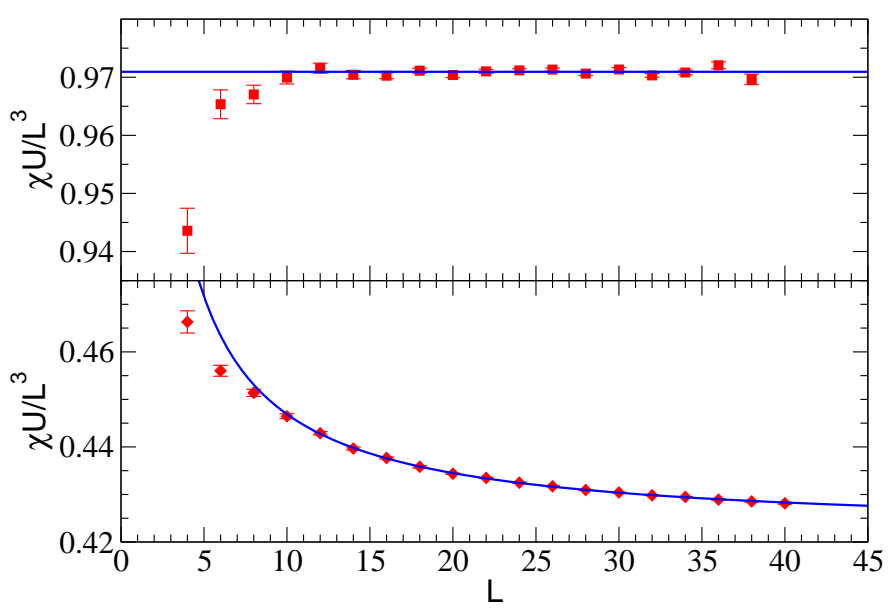

Figure 2: Plot of the chiral susceptibility $\chi$ as a function of $L$ at $U=\infty$ for the $Z_{2}$ (top) and $U$ (1) (bottom) models. The solid curve in the top graph is a fit to a constant for $L \geq 16$, while in the bottom graph it is a fit to the finite size scaling form for $L \geq 10$ obtained from chiral perturbation theory.

important to distinguish this feature in our results. For this purpose we have computed the chiral condensate susceptibility,

$$
\chi=\frac{1}{L^{3}} \sum_{x, y}\left\langle\bar{\chi}_{x} \chi_{x} \bar{\chi}_{y} \chi_{y}\right\rangle
$$

as a function of the lattice size $L$ at $U=\infty$ where the effects must be clearly visible. At infinite coupling our models can be mapped into a statistical model of closed packed dimers and can be updated very efficiently using worm algorithms [19]. Our results are shown in Fig. 2. The chiral condensate susceptibility is non-zero in the thermodynamic limit as expected. Further, the finite size effects are indeed enhanced in the $U(1)$ invariant model due to the presence of massless Goldstone bosons and fit well to the leading order chiral perturbation theory form [20]. On the other hand the data for the $Z_{2}$ model is almost flat.

We study the properties of the quantum critical point through the chiral susceptibility (3.1) and the fermion correlation function ratio $R_{f}=C_{F}(L / 2-1) / C_{F}(1)$ where

$$
C_{F}(d)=\frac{1}{3} \sum_{\alpha=1}^{3}\left\langle\chi_{x} \bar{\chi}_{x+d \hat{\alpha}}\right\rangle .
$$

In the above expression $x$ is the origin or any translation of it by multiples of two lattice spacings in each direction, and $\hat{\alpha}$ is a unit vector along each of the three directions. With massless fermions, in the vicinity of $U_{c}$, we expect $\chi / L^{2-\eta}$ and $R_{f} L^{2+\eta_{\psi}}$ to be an analytic function of $\left(U-U_{c}\right) L^{1 / v}$. In particular, at $U=U_{c}$ they will be constants independent of the volume. Our data fits well to this form if we keep the first five terms in the Taylor expansion. Plots of our data along with fits are shown in Fig. 3 and the critical exponents are tabulated in Table. 1.

\section{Conclusions}

It is interesting that both $Z_{2}$ and $U(1)$ symmetric models have very similar exponents, although 

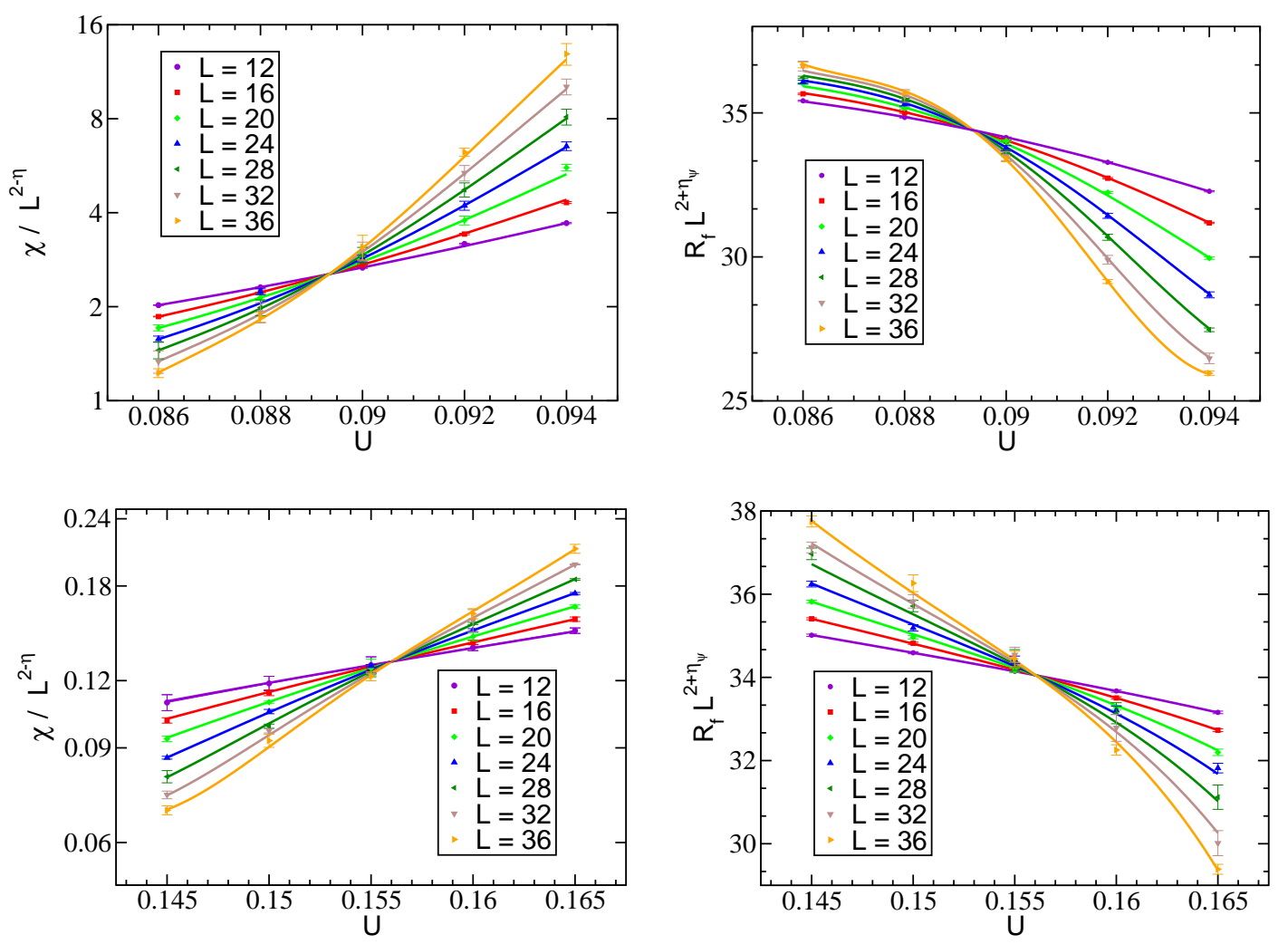

Figure 3: Plots of $\chi / L^{2-\eta}$ and $R_{f} L^{2+\eta_{\psi}}$ as a function of $U$ for $L$ from 12 to 36. The solid lines show the combined fit which gives $U_{c}=0.0893(1), v=0.83(1), \eta=0.62(1)$ and $\eta_{\psi}=0.38(1)$ in the $Z_{2}$ case (top row) and $U_{c}=0.1560(4), v=0.82(2), \eta=0.63(2), \eta_{\psi}=0.37(1)$ in the $U(1)$ case (bottom row).

\begin{tabular}{ccccc}
\hline \hline$U_{c}$ & $v$ & $\eta$ & $\eta_{\psi}$ & $\chi^{2} /$ d.o.f \\
\hline $0.0893(1)$ & $0.83(1)$ & $0.62(1)$ & $0.38(1)$ & 1.8 \\
\hline $0.1560(4)$ & $0.82(2)$ & $0.63(2)$ & $0.37(1)$ & 0.88 \\
\hline \hline
\end{tabular}

Table 1: Results for the critical exponents obtained from a combined fit of the data in the critical region in the $Z_{2}$ invariant model (top row) and $U(1)$ invariant model (bottom row).

their large $U$ phases are clearly different. Further, the critical exponents in the $S U(2) \times U(1)$ symmetric lattice Gross-Neveu model obtained here, are consistent with those in the lattice Thirring model [14]. Thus, the folk lore that the two models belong to different universality classes is incorrect. This is reassuring since the two models have the same lattice symmetries. We have also studied another $S U(2) \times U(1)$ invariant model with $U_{\mathbb{L}}=U_{\mathbb{B}}=U, U_{\mathbb{F}}=U_{\mathbb{N}}=0$ and found that the critical exponents are again consistent with the above values [21]. Combining all data from the three different studies and performing a single combined fit we estimate the critical exponents in the $S U(2) \times U(1)$ symmetric single staggered flavor lattice models to be $v=0.849(8), \eta=0.633(8)$ and $\eta_{\psi}=0.373$ (3). Finally, we believe the critical exponents obtained in Ref. [9] are incorrect 
since the model studied has the same lattice symmetries as our $S U(2) \times Z_{2}$ model. Hence the critical exponents obtained in Ref. [9] should have been consistent with our results. Perhaps the reason for this is that the earlier study ignored the sign problem.

\section{Acknowledgments}

We thank H. Gies, S. Hands, L. Janssen, B. Rosenstein and C. Strouthos for helping us understand this complex subject slightly better. This work was supported in part by the Department of Energy grants DE-FG02-05ER41368 and DE-FG02-00ER41132.

\section{References}

[1] T. Appelquist, J. Terning and L. C. R. Wijewardhana, Phys. Rev. Lett. 77, 1214 (1996).

[2] T. Appelquist, G. T. Fleming, M. F. Lin, E. T. Neil and D. A. Schaich, Phys. Rev. D 84, 054501 (2011).

[3] B. Rosenstein, B. Warr, and S. H. Park, Phys.Rept. 205, 59 (1991).

[4] J. Braun, H. Gies and D. D. Scherer, Phys. Rev. D 83, 085012 (2011).

[5] A. H.Castro Neto, F. Guinea, N. M. R. Peres, K. S. Novoselov, and A. K. Geim, Rev.Mod. Phys. 81, 109, 2009.

[6] V. N. Kotov, B. Uchoa, V. M. Pereira, A. H. C. Neto and F. Guinea, Rev. Mod. Phys. 84, 1067 (2012).

[7] S. Hands, A. Kocic and J. B. Kogut, Annals Phys. 224, 29 (1993).

[8] F. Hofling, C. Nowak and C. Wetterich, Phys. Rev. B 66, 205111 (2002).

[9] L. Karkkainen, R. Lacaze, P. Lacock and B. Petersson, Nucl. Phys. B 415, 781 (1994) [Erratum-ibid. B 438, 650 (1995)].

[10] S. Chandrasekharan and A. Li, Phys. Rev. D 85, 091502 (2012).

[11] L. Janssen and H. Gies, Phys. Rev. D 86, 105007 (2012).

[12] L. Del Debbio et al. [UKQCD Collaboration], Nucl. Phys. B 502, 269 (1997) [hep-lat/9701016].

[13] I. M. Barbour et al. [UKQCD Collaboration], Phys. Rev. D 58, 074507 (1998).

[14] S. Chandrasekharan and A. Li, Phys. Rev. Lett. 108, 140404 (2012).

[15] S. Christofi and C. Strouthos, JHEP 0705, 088 (2007).

[16] S. Chandrasekharan, Eur. Phys. J. A 49, 90 (2013).

[17] H. S. Sharatchandra, H. J. Thun and P. Weisz, Nucl. Phys. B 192, 205 (1981).

[18] S. Catterall, R. Galvez, J. Hubisz, D. Mehta and A. Veernala, Phys. Rev. D 86, 034502 (2012).

[19] N. Prokof'ev and B. Svistunov, Phys. Rev. Lett. 87, 160601 (2001).

[20] P. Hasenfratz and H. Leutwyler, Nucl. Phys. B 343, 241 (1990).

[21] A. Li, J. Phys. Conf. Ser. 432, 012024 (2013). 